\title{
Bi- or Ter-pyridine Tris-Substituted Benzenes as Electron-Transporting Materials for Organic Light-Emitting Devices
}

Musubu Ichikawa ${ }^{1,2, *}$, Kenta Wakabayashi ${ }^{1}$, Shuichi Hayashi ${ }^{3}$, Norimasa Yokoyama ${ }^{3}$, Toshiki Koyama $^{1}$, Yoshio Taniguchi ${ }^{4}$

${ }^{1}$ Functional Polymer Science Course, Faculty of Textile Science and Technology, Shinshu University, 3-15-1 Tokida, Ueda City, Nagano 386-8567, Japan

${ }^{2}$ Presto, Japan Science and Technology Agency (JST), 4-8-1 Honcho, Kawaguchi, Saitama 332-0012, Japan

${ }^{3}$ Hodogaya Chemical Co., Ltd., 45 Miyukigaoka, Tsukuba, Ibaraki 305-0841, Japan

${ }^{4}$ Faculty of Textile Science and Technology, Shinshu University, 3-15-1 Tokita, Ueda, Nagano 386-8567, Japan

\begin{abstract}
We demonstrated that 1,3,5-tris([2',2']bipyridin-6'-yl)benzene (BpyB) and 1,3,5tris $\left(\left[2^{\prime}, 2^{\prime \prime}, 6^{\prime \prime}, 2^{\prime \prime \prime}\right]\right.$ terpyridin-6'-yl)benzene (TpyB) are good electron-transport (ET) layer materials for organic light-emitting devices (OLEDs). The new materials exhibit high electron mobilities of around $10^{-4} \mathrm{~cm}^{2} \mathrm{~V}^{-1} \mathrm{~s}^{-1}$, and OLEDs comprising the materials operate at lower voltage than the OLEDs with tris(8-hydroxyquinolinato) aluminum (Alq) as an ET material. The new materials are also useful for phosphorescent OLEDs in blue-, green-, and red-light-emitting devices, where they function as both electron-transporting and hole-blocking materials. Phosphorescent OLEDs with the new materials operate at lower voltage (while maintaining comparable external quantum efficiencies of electroluminescence) than does the corresponding reference device with bathocuproine/Alq as electron-transporting and hole-blocking layers.
\end{abstract}

Keywords: Electron transporting; Hole blocking; Electroluminescent device; OLED; Phosphorescent device; Mobility; Pyridine; Bipyridine; Terpyridine

*Corresponding author. Tel: +81-268-21-5498; Fax: +81-268-21-5413; E-mail: musubu@shinshu-u.ac.jp (M. Ichikawa) 
1. Introduction

Organic light-emitting devices (OLEDs) are of interest for potential application in various fields such as flat televisions, mobile displays, lights, and optical communication light sources. OLEDs generally comprise functionally divided organic multilayers-for example, holetransporting (HT), emissive, and electron-transporting (ET) layers. [1, 2] In the last decade, many kinds of amorphous molecular semiconductor materials, [3, 4] HT materials, [5-12] and ET materials, [13-23] have been proposed. Among these, HT molecular semiconducting materials have become practical due to their high charge-carrier mobility and excellent operational durability, but few reports exist to date on ET organic semiconducting amorphous materials that perform well in terms of high-speed transport of electrons, easy injection of electrons from the cathode, and good operational durability. [16] Tris (8-hydroxyquinolinato) aluminum(III) (Alq) was used in the first bright OLED reported by Tang et al. in 1987 [1]; it is still conventionally used as an ET material, despite its slow electron mobility, [24] because of its relatively good operational durability. However, as a reflection of the imbalanced performance between ET and HT materials, electron-feeding from the cathode into an emissive layer controls device characteristics. As a result, the development of efficient ET organic amorphous semiconducting materials remains an important challenge.

ET materials with high electron mobilities provide some advantages, including lower operating voltage and lower power consumption. Moreover, if ET materials have wide band gaps - that is, deep highest occupied molecular orbitals (HOMOs) - they can also work as holeblocking (HB) materials. Hole-blocking is desirable because complete confinement of holes in emissive layers by the HB layer raises the quantum efficiency (QE) of electroluminescence (EL). Furthermore, when considering phosphorescent OLEDs (PHOLEDs) that can theoretically provide the $100 \%$ internal electron-photon conversion efficiency theoretically [25], a complete confinement for triplet exciton energies of phosphorescent light-emitters also becomes important to attain extremely high QE.

We have frequently reported on ET materials with high electron mobilities based on one molecular design concept: hybridizing bipyridine with common ET units such as oxadiazole or triazole. [26-28] Can we now make good ET materials based on bipyridine only? Bathophenanthroline, a common good ET material, is similar in structure to bipyridine. Kido et al. recently reported that tris-pyridine-substituted triphenylbenzenes exhibit very good electron- 
transport properties, with electron mobilities as high as $7.9 \times 10^{-3} \mathrm{~cm}^{2} \mathrm{~V}^{-1} \mathrm{~s}^{-1}$. [29] As a result, we can assume that the tris-bipyridine-substituted benzene (BpyB) and tris-terpyridine-substituted benzene (TpyB) examined in this study will be good ET-layer materials for OLEDs. As described herein, OLEDs with these new materials clearly operate at lower voltage than do OLEDs with Alq, a conventional ET material. The new materials are also useful for phosphorescent OLEDs, where they work not only as ET but also as HB materials.

\section{Experimental}

\subsection{Materials}

Figure 1 shows chemical structures of the newly developed materials BpyB and TpyB, synthesized from bipyridine or terpyridine bromides with tris(pinacolboryl) benzene by SuzukiMiyaura coupling reaction. BpyB and TpyB were purified, before evaluation and measurements, by temperature-gradient sublimation in a flow stream of pure Ar gas. Other OLED materials were obtained from industrial companies as sublimation grades and used without further purification.

\subsection{Synthesis}

\section{6-Bromo[2,2']bipyridine (Br-Bpy)}

Br-Bpy was synthesized from 2,6-dibromopyridine (11.8 g, $52.8 \mathrm{mmol})$ and 2-pyridyl zinc bromide $(55.0 \mathrm{mmol})$ that was contained in $110 \mathrm{ml}$ of its tetrahydrofuran (THF) solution (0.5 M) using $50 \mathrm{ml}$ of THF as additional solvent and tetrakis(triphenylphosphine) $\operatorname{Pd}(0)(1.7 \mathrm{~g}, 2.5$ $\mathrm{mmol}$ ) as catalyst. With the reaction solution kept below $10^{\circ} \mathrm{C}$ with an ice bath, the 2-pyridyl zinc bromide solution was added dropwise. The ice bath was removed and the solution was stirred continuously for $48 \mathrm{~h}$. Then, $10-\mathrm{wt} \%$ ethylenediamine-N,N,N',N'-tetraacetic acid, disodium salt (EDTA $2 \mathrm{Na}$ ) aqueous solution $(500 \mathrm{ml})$ was added in small steps. Chloroform extraction and distillation of the solvent gave a crude brown powder. Purification by silica gel chromatography with a toluene/ethyl acetate mixture as eluent yielded Br-Bpy as white powder ( $7.0 \mathrm{~g} ; 54 \%$ yield). $\delta_{\mathrm{H}}\left(600 \mathrm{MHz}, \mathrm{CDCl}_{3}\right): 8.67(\mathrm{~d}, 1 \mathrm{H}, \mathrm{J} 4.8 \mathrm{~Hz}), 8.41$ (d, 1H, J $\left.7.8 \mathrm{~Hz}\right), 8.38$ (d, 1H, J 8.4), 7.82 (dd, 1H, J 8.7 Hz), 7.67 (dd, 1H, J 7.8 Hz), 7.49 (d, 1H, J 7.2 Hz), 7.32 (dd, 1H, J 6.3 Hz). 
6-Bromo[2,2',6',2"]terpyridine (Br-Tpy)

Br-Tpy was synthesized from 6,6'-dibromo[2,2']bipyridine (10.0 g, $31.4 \mathrm{mmol})$ essentially as described for Br-Bpy to yield a white powder $\left(5.2 \mathrm{~g} ; 52.4 \%\right.$ yield). $\delta_{\mathrm{H}}(600 \mathrm{MHz}$, $\mathrm{CDCl}_{3}$ ): 8.70 (d, 1H, J 4.8 Hz), 8.58 (d, 1H, J $\left.6.6 \mathrm{~Hz}\right), 8.57$ (d, 1H, J 9.0 Hz), 8.46 (m, 2H), 7.95 (dd, 1H, J 7.8 Hz), 7.85 (dd, 1H, J 6.0 Hz, J 7.8 Hz), 7.70 (dd, 1H, J 8.1 Hz), 7.50 (d, 1H, J 7.2 Hz) $7.34(\mathrm{~m}, 1 \mathrm{H})$.

\section{1,3,5-Tris(pinacorato boryl)benzene $\left((\mathrm{PinB})_{3} \mathrm{~B}\right)$}

$(\mathrm{PinB})_{3} \mathrm{~B}$ was synthesized by Suzuki-Miyaura coupling reaction using 1,3,5tribromobenzene $(8.6 \mathrm{~g}, 27.3 \mathrm{mmol})$, bis(pinacorato)diboron $(25.0 \mathrm{~g}, 98.4 \mathrm{mmol})$, potassium acetate (24.1 g, $245.7 \mathrm{mmol})$, and [1,1'-bis(diphenylphosphino)ferrocene] dichloropalladium(II) as catalyst in dimethyl sulfoxide. The reaction mixture was refluxed for $21 \mathrm{~h}$, and then cooled to room temperature. Pure water $(1000 \mathrm{ml})$ was added and the mixture was stirred for $30 \mathrm{~min}$ and filtered to remove precipitates. Addition of methanol gave a crude white crystalline powder, which was dissolved in ethyl acetate, filtered to remove the catalyst, and distilled to yield pure (PinB) ${ }_{3} \mathrm{~B}\left(7.1 \mathrm{~g} ; 57 \%\right.$ yield). $\delta_{\mathrm{H}}\left(600 \mathrm{MHz}, \mathrm{CDCl}_{3}\right): 8.37$ (s, 3H), 1.33 (s, 36H).

\section{1,3,5-Tris $\left(\left[2^{\prime}, 2^{\prime \prime}\right]\right.$ bipyridin-6'-yl)benzene (BpyB)}

BpyB was synthesized from (PinB $)_{3} \mathrm{~B}(2.5 \mathrm{~g}, 5.5 \mathrm{mmol})$ and Br-Bpy $(3.8 \mathrm{~g}, 15.0 \mathrm{mmol})$ by Suzuki-Miyaura coupling reaction using $1 \mathrm{M} \mathrm{K}_{2} \mathrm{CO}_{3}$ aqueous solution (32.4 ml, $32.4 \mathrm{mmol}$ ) as base, tetrakis(trisphenylphosphine)palladium(0) $(0.32 \mathrm{~g}, 0.28 \mathrm{mmol})$ as catalyst, and $135 \mathrm{ml}$ of a 4/1 ethanol/toluene mixture as solvent. The reaction mixture was refluxed with stirring for $33 \mathrm{~h}$, and then cooled to room temperature. Pure water $(500 \mathrm{ml})$ was added and the mixture was stirred for $30 \mathrm{~min}$. Extraction by chloroform, drying with $\mathrm{MgSO}_{4}$, filtration to remove precipitates, and distillation to remove the chloroform gave a crude brown powder. Purification by silica gel column chromatography with a $1 / 3$ chloroform/hexane mixture as eluent yielded BpyB as a white crystalline powder $\left(1.1 \mathrm{~g} ; 41 \%\right.$ yield). $\delta_{\mathrm{H}}\left(600 \mathrm{MHz}, \mathrm{CDCl}_{3}\right): 9.01(\mathrm{~s}, 3 \mathrm{H}), 8.76(\mathrm{~d}, 3 \mathrm{H}, \mathrm{J} 7.2 \mathrm{~Hz})$, 8.74 (d, 3H, J 4.8 Hz), 8.47 (d, 3H, J 7.2 Hz), 8.01 (dd, 3H, J 8.7 Hz), 7.98 (d, 3H, J 7.8 Hz), 7.88 $(\mathrm{dd}, 3 \mathrm{H}, \mathrm{J} 7.8 \mathrm{~Hz}), 7.36(\mathrm{dd}, 3 \mathrm{H}, \mathrm{J} 6.3 \mathrm{~Hz}) . \delta_{\mathrm{C}}\left(150 \mathrm{MHz}, \mathrm{CDCl}_{3}\right): 156.43,156.31,155.90$, $149.14,140.43,137.82,136.82,126.25,123.78,121.37,120.65,119.65$ 


\section{1,3,5-Tris $\left(\left[2^{\prime}, 2^{\prime \prime}, 6^{\prime \prime}, 2^{\prime \prime \prime}\right]\right.$ terpyridin-6'-yl)benzene (TpyB)}

TpyB was synthesized by Suzuki-Miyaura coupling reaction using (PinB) ${ }_{3} \mathrm{~B}(2.4 \mathrm{~g}, 5.26$ mmol) and Br-Tpy (5.0 g, $16.0 \mathrm{mmol})$ essentially as described for BpyB using $1 \mathrm{M} \mathrm{K}_{2} \mathrm{CO}_{3}$ aqueous solution $(32.3 \mathrm{~g}, 32.4 \mathrm{mmol})$, tetrakis(trisphenylphosphine)palladium(0) (0.32 g, 0.28 $\mathrm{mmol}$ ), and $150 \mathrm{ml}$ of a $4 / 1$ ethanol/toluene mixture. The reaction mixture was refluxed for $3 \mathrm{~h}$, cooled, and then filtered. Recrystallization from $o$-dichlorobenzene solution yielded TpyB as a white powder $(2.7 \mathrm{~g} ; 67 \%$ yield $) . \delta_{\mathrm{H}}\left(600 \mathrm{MHz}, \mathrm{CDCl}_{3}\right): 9.06(\mathrm{~s}, 3 \mathrm{H}), 8.81(\mathrm{~d}, 3 \mathrm{H}, \mathrm{J} 7.8 \mathrm{~Hz})$, 8.73(d, 3H, J 3 Hz), 8.68 (d, 3H, J 8.4Hz), 8.67 (d, 3H, 7.8 Hz), 8.50 (d, 3H, J 8.4 Hz), 8.03 (m, 9H), 7.89 (dd, 3H, J $7.8 \mathrm{~Hz}), 7.35$ (d, 3H, J 7.8 Hz). $\delta_{\mathrm{C}}\left(150 \mathrm{MHz}, \mathrm{CDCl}_{3}\right): 156.21,155.85$, $155.54,155.30,149.15,140.28,137.77,136.87,130.51,127.70,126.17,123.75,121.26,121.21$, $121.05,120.60,119.69$.

\subsection{Device Fabrication and Measurements}

All OLEDs were fabricated on 150-nm-thick layers of indium-tin oxide (ITO) that were commercially precoated onto glass substrates with a sheet resistance of $14 \Omega /$ sq. The solventcleaned ITO surface was treated with $\mathrm{O}_{2}$ plasma for $5 \mathrm{~min}$ and then the substrate was immediately loaded into a high-vacuum chamber (base pressure below $\sim 6 \times 10^{-4} \mathrm{~Pa}$ ) where organic layers, 0.5-nm-thick LiF, and 200-nm-thick aluminum cathode layers were deposited by thermal evaporation. Deposition rates were $0.6 \AA / \mathrm{s}$ for organic materials, $0.1 \AA / \mathrm{s}$ for LiF, and 6 $\AA / \mathrm{s}$ for Al. The current density-applied voltage-luminance $(J-V-L)$ characteristics of the OLEDs were measured with a commercial OLED-characteristics measurement system (Precise Gauge EL1003) and a source meter (Keithley 2400). Electroluminescence (EL) spectra were recorded simultaneously with the EL1003 during $J-V-L$ measurements.

${ }^{1} \mathrm{H}$ - and ${ }^{13} \mathrm{C}-\mathrm{NMR}$ spectra were recorded on an FT-NMR spectrometer (JEOL JNMECA600). Thermal analyses were performed on a differential scanning calorimeter (Seiko Instruments DSC-6200) at a heating rate of $10^{\circ} \mathrm{C} / \mathrm{min}$ under $\mathrm{N}_{2}$ gas. Ultraviolet (UV) and visible absorption spectra were recorded with a spectrophotometer (Shimadzu UV-3150); photoluminescence (fluorescence and phosphorescence) spectra were recorded with a spectrofluorometer (Horiba FluoroMax4P). HOMO energy was determined with a photoelectron 
emission yield spectrometer (Riken Keiki AC-3), and the HOMO energy was defined as being equal to the ionization potential measured by photoelectron emission spectroscopy. Optical band gaps were determined by the spectral onset of each UV-visible absorption spectrum; the lowest unoccupied molecular orbital (LUMO) energy was then estimated to be the difference between the HOMO energy and the optical bandgap. Spectroscopic measurements were conducted with thin films prepared by thermal evaporation on quartz substrates.

Electron mobility was measured by conventional time-of-flight (TOF) techniques. The excitation light was a 500-ps-duration optical pulse from an $\mathrm{N}_{2}$ gas laser $(\lambda=337 \mathrm{~nm}$, Usho Optical Systems KEN-150). Test samples were prepared by thermal evaporation in vacuum and encapsulated with fresh desiccant under a highly inert atmosphere of $\mathrm{N}_{2}$ at the dew point of almost $-60^{\circ} \mathrm{C}$ and $\mathrm{O}_{2}$ concentration of $<10 \mathrm{ppm}$. A 100 -nm-thick fullerene $\mathrm{C}_{60}$ layer was used as a charge-generation layer for optical excitation. [27]

Computational chemistry was conducted with commercial software (Wavefunction Spartan 06W) on a personal computer with a dual-core processer. Density function theory (DFT) was used to determine conformations, with a B3LYP hybrid functional and a $6-311 \mathrm{G}^{*}$ basis set for determining optimized geometries and estimating activation energies for conformation change. During the estimation of activation energies, we constrained the dihedral angle that was responsible for the conformation change and conducted each geometry optimization with the dihedral constraint at several angles.

\section{Results and Discussion}

\subsection{Thermal and Electronic Properties}

Figure 2 shows differential scanning calorimetry (DSC) curves for BpyB and TpyB. During the first heating procedure, endothermic peaks due to melting are observed for the two powders at $240^{\circ} \mathrm{C}$ and $305^{\circ} \mathrm{C}$, respectively. For $\mathrm{BpyB}$, during the second heating procedure, a baseline shift due to glass transition and an exothermic peak at $146^{\circ} \mathrm{C}$ due to recrystallization are observed; the glass transition temperature $T \mathrm{~g}$ is $75^{\circ} \mathrm{C}$. For TpyB, during the second heating procedure, the same transitions occur: recrystallization at $166^{\circ} \mathrm{C}$ and $\mathrm{Tg}$ at $110^{\circ} \mathrm{C}$. These results confirm that both powders can form stable glassy thin films by vacuum evaporation. $\mathrm{Tg}$ is higher for TpyB $\left(110^{\circ} \mathrm{C}\right)$ than for NPB $\left(94^{\circ} \mathrm{C}\right)$, a practical HT material (Fig. 5). 
Figure 3(a) shows the optimized geometry for BpyB, obtained by means of computational chemistry. The geometry shows C3 symmetry and resembles a three-blade propeller. The dihedral angle between the center phenyl ring and the adjacent pyridine ring is $23^{\circ}$ because of steric hindrance between the two hydrogen atoms at the ortho position of each ring. In contrast, the pyridine units are coplanar. These angles are reasonable from the viewpoint of general chemistry. Figure $3(\mathrm{~b})$ shows a bipyridyl wing of BpyB rotated $180^{\circ}$ around the single bond that connects the bipyridine unit with the center phenyl ring, significantly changing the molecular geometry. Note that the energy of this rotated geometry is the third lowest and the energy difference $\Delta E$ between the two conformers is estimated to be only $1.77 \mathrm{kJmol}^{-1}$. Such rotation is known to occur easily in the case of conformation with a single bond that links two aromatic rings. Figure 3(c) shows the energy profile for dihedral rotation. Through computational chemistry, we estimate the activation energy to be $15.5 \mathrm{kJmol}^{-1}$, only $\sim 6$ times larger than the thermal energy at room temperature $\left(2.48 \mathrm{kJmol}^{-1}\right)$. This result indicates that conformation change between the two conformers occurs frequently, and a population of the higher energy conformer must be defined by the Boltzmann distribution with the energy difference $\Delta E$. The exact population must depend on the energy distributions of all the conformers of BpyB, but this small $\Delta E$ suggests that a large number of BpyB molecules exist as conformers other than the lowest energy one, and thus thin films of BpyB are composed of several conformers. We believe that this is one reason why BpyB displays an amorphous nature. [27]

Figure 4(a) shows UV-visible absorption and fluorescence spectra of neat thin films of each material. The absorption maxima are at $274 \mathrm{~nm}$ for BpyB and $293 \mathrm{~nm}$ for TpyB, and both materials emit bright UV fluorescence. Optical interference by the sample thin film causes broad absorption around $500-600 \mathrm{~nm}$. The band gaps $(E \mathrm{~g}=3.60 \mathrm{eV})$ for both BpyB and TpyB from the absorption spectra are clearly wide.

Figure 4(b) shows phosphorescent spectra of dilute 2-methyltetrahydrofuran (meTHF) solutions of BpyB and TpyB at liquid-nitrogen temperature $(77 \mathrm{~K})$. We determined the triplet bandgap energy $E \mathrm{~g}^{\mathrm{T}}$ from an apparent peak at the shortest wavelength, indicated by an arrow in the figure; for BpyB, $E \mathrm{~g}^{\mathrm{T}}=2.77 \mathrm{eV}$ and for TpyB, $E \mathrm{~g}^{\mathrm{T}}=2.79 \mathrm{eV}$. For BpyB, the LUMO level $(2.85 \mathrm{eV})$ in neat solid is low enough to easily accept electrons from a cathode metal. For TpyB, the LUMO level is deeper $(3.00 \mathrm{eV})$ due to three additional pyridine units, implying easier injections of electrons from a cathode metal. In addition, for both materials, the HOMO levels 
(6.45 eV for BpyB and $6.60 \mathrm{eV}$ for TpyB) are sufficiently deep to suppress hole leakages from the emissive layer, and hole confinement in the emissive layer leads to high QE of EL. As a result, BpyB and TpyB can be used as efficient ET and HB materials. The thermal and electronic properties of BpyB and TpyB are summarized in Table 1.

\subsection{OLED properties}

Figure 5 shows the current density-applied voltage $(J-V)$ characteristics of OLEDs with BpyB or TpyB as an ET material as well as of a reference device without the new materials. Figure 6 shows the structure of the associated OLED and the various chemicals used in it. OLEDs with the new materials perform better (operate at lower voltage) than a reference OLED with Alq. Therefore, we conclude that we can make a good ET material with bipyridine and/or terpyridine only. The BpyB device shows almost the same luminous efficiency $\left(4.0 \mathrm{cdA}^{-1}\right)$ as does the reference device with Alq. The TpyB device shows even higher luminous efficiency (5.0 $\mathrm{cdA}^{-1}$ ), probably due to the deeper HOMO of TpyB, which is effective for preventing hole leakages from the emissive layer. As noted earlier, hole-blocking is important for attaining high QE of EL. While we observed no increase in efficiency for BpyB, the lower operation voltage of the device with BpyB implies high electron mobility of BpyB, and the high mobility might move the electron-hole recombination zone for the NPB/Alq interface. The move of the recombination zone is probably a reason no increase of the QE because a very recent report indicated that an interaction between NPB and Alq apparently reduces the luminescence efficiency of Alq [30]. We believe that BpyB should also have HB properties because of its sufficiently deep HOMO.

Because BpyB and TpyB perform well not only as ET materials but also as HB materials, we investigated their use as double-function ET/HB materials. Recent highly efficient phosphorescent OLEDs have both an HB layer and an ET layer. In particular, an HB layer is important for obtaining high QE because hole leakage seriously reduces efficiency. Moreover, it is more important to consider triplet energy dissipation from the emissive layer to the holeblocking layer for attaining high QE. At present, only a few materials are used as HB materials for phosphorescent OLEDs, including bathocuproine (BCP) and bis (2-methyl-8-quinolinolato) ( $p$-phenylphenolato) aluminum (BAlq).

Figure 7 shows the $J-V$ characteristics of phosphorescent OLEDs with BpyB or TpyB as a 
double-function ET/HB material as well as of a reference OLED. Figure 8 shows the structure of the associated phosphorescent OLED and the various chemicals used in it. Phosphorescent OLEDs with both new materials perform better (operate at much lower voltage) than a conventional double-layer reference OLED comprising BCP and Alq layers. [31] Since devices with both new materials show high external QE (almost 10\%), BpyB and TpyB are both useful as double-function ET/HB materials for phosphorescent OLEDs. As shown in Table 1, the $E \mathrm{~g}^{\mathrm{T}}$ for each new material is so large that no triplet energy leakage from the Ir-complex phosphorescent light emitter occurs.

The new materials appear to be effective even for blue-light-emitting Ir complex because the $E \mathrm{~g}^{\mathrm{T}} \mathrm{S}$ of the materials are sufficiently large enough to prevent leakages of the triplet energy in blue-lightf-emitting $\quad$ Ir complexes such as $\operatorname{Ir}(\mathrm{III})$ bis(2-(4,6-difluorophenyl)pyridinato$\mathrm{N}, \mathrm{C}^{2}$ )picolinate (FIrpic) of which $E \mathrm{gT}$ is $2.62 \mathrm{eV}$ [32]. This is clearly evident from Figure 9, which shows the $J$ - $V$-external QE characteristics of blue- and red-light-emitting PHOLEDs with the corresponding dopants ( $\mathrm{Ir}(\mathrm{III})$ bis(2-(4,6-difluorophenyl)pyridinato-N, $\left.\mathrm{C}^{2}\right)$ picolinate (FIrpic) for blue and $\operatorname{Ir}(\mathrm{III})$ bis(2-(2'-benzothienyl)pyridinato-N, $\left.\mathrm{C}^{3}\right)$ acetylacetonate (BtpIracac) for red. Note that we fabricated those devices based on the same structure shown in Fig. 8 except for the dopants. Fig. 9 clearly shows that using the new materials reduce operation voltages in both colors. In addition, as one can see from Fig. 9, the devices comprising the new materials in both luminescent colors showed comparable external QEs to the corresponding reference devices with BCP and Alq. However, a slight but obvious difference in the QEs between the BpyB and TpyB devices could be observed in the blue devices. Because both $E \mathrm{~g}^{\mathrm{T}} \mathrm{S}$ are almost the same, the difference probably results from a change of the recombination zone due to higher electron mobility of BpyB-the triplet energies of FIrpic and also maybe CBP possibly leak to NPB.. The lower operation voltages and the comparable external QEs indicate that both BpyB and TpyB are also useful for phosphorescent OLEDs in both colors. Further optimization of device structures, including changes of host and other materials, should lead to higher external QEs at low operating voltage.

\subsection{Electron Mobility}

Finally, we would like to briefly discuss electron mobility in the new materials. Figure 10 
shows the transient electron photocurrent waveform with double logarithmic scale, obtained by TOF measurement, for the BpyB film. Although each photocurrent waveform was not clear nondispersive, we could clearly see that the transits of photogenerated charge carriers-electrons in this case become faster with increasing applied voltages because the photocurrents decayed faster with the increaseing. In accordance with the conventional method to determine a transit time $\left(t_{\mathrm{T}}\right)$ for dispersive transportations, we drew the two auxiliary straight lines as shown in the figure and determined the $t_{\mathrm{T}}$ at the crossing point of the auxiliary lines. Note that the two slopes of the two auxiliary lines for each photocurrent are almost the same as each other, and this also endorses that the observed photocurrents are due to drift motions in the film. If we take electron mobility $\mu$ to be

$$
\mu=D d /\left(t_{T} V\right)
$$

where $D$ is the distance between the two electrodes $(4.0 \mu \mathrm{m}), d$ is the thickness of the prepared BpyB layer $(3.9 \mu \mathrm{m})$, and $V$ is the applied voltage, we can determine, for example, that $\mu=2.6 \times$ $10^{-4} \mathrm{~cm}^{2} \mathrm{~V}^{-1} \mathrm{~s}^{-1}$ at the electric field $E=V / D=1.13 \times 10^{6} \mathrm{Vcm}^{-1}$. This mobility is two orders of magnitude higher than that for Alq $\left(1.3 \times 10^{-6} \mathrm{~cm}^{2} \mathrm{~V}^{-1} \mathrm{~s}^{-1}\right)$ at approximately the same electric field. [33] This high mobility probably accounts for the better $J-V$ curve for the OLED with BpyB than for the OLED with the corresponding reference.

Figure 11 shows electron mobility as a function of the square root of the applied electric field for several materials, including BpyB and TpyB. Both BpyB and TpyB show mobility that is at least an order of magnitude higher than that of Alq. This high mobility is comparable to that of a representative ET material with high electron mobility: a silole derivative, 2,5-bis $\left(6^{\prime}-\left(2^{\prime}, 2^{\prime \prime}-\right.\right.$ bipyridine))-1,1-dimethyl-3,4-diphenylsilole (PyPySiPyPy). [33, 34] The mobility was relatively high but lower than that $\left(1.0 \times 10^{-3} \mathrm{~cm}^{2} \mathrm{~V}^{-1} \mathrm{~s}^{-1}\right.$ at $\left.6.4 \times 10^{5} \mathrm{Vcm}^{-1}\right)$ of tris-pyridyl substituted 1,3,5-triphenylbenzene (TmPyPB) reported by Kido et al [29] of which structure was similar to BpyB, and we are unsure about the reasons for the difference at present. In addition, mobility is lower for TpyB than for BpyB. Although we are also not sure at present about the reason, the lower mobility reflects the higher operating voltages for TpyB devices than for BpyB devices, as shown in all of the figures showing $J-V$ characteristics.

\section{Conclusion}


We demonstrated that tris-bipyridine-substituted benzene (BpyB) and tris-terpyridinesubstituted benzene (TpyB) are good ET-layer materials for OLEDs. Electron mobilities for these new materials $\left(\sim 10^{-4} \mathrm{~cm}^{2} / \mathrm{Vs}\right)$ are much higher than that for Alq and almost the same as that for conventional high-mobility ET materials such as bathophenanthroline and silole derivatives. OLEDs with these new materials operate at lower voltages than does an OLED with Alq as an ET material. The new materials are also useful for phosphorescent OLEDs in blue-, green-, and red-light-emitting devices, where they work as both electron-transporting and hole-blocking materials. Phosphorescent OLEDs with the new materials operate at lower voltage (while maintaining comparable external quantum efficiencies) than do the corresponding reference devices with $\mathrm{BCP} / \mathrm{Alq}$ as electron-transporting and hole-blocking layers.

\section{Acknowledgements}

We thank Dr. Y. Nakajima of Riken Keiki for measuring the ionization potential of compounds with their photoelectron yield spectroscopic equipment (Riken Keiki AC-3). This work was supported by Regional Innovation Cluster Program of Nagano, granted by the Ministry of Education, Culture, Sports, Science, and Technology, Japan. This article is dedicated to the first principal of the Faculty of Textile Science and Technology, Shinshu University, Chotaro Harizuka, on the occasion of the 100th anniversary of the faculty. 


\section{References}

[1] C. W. Tang, S. A. VanSlyke, Appl. Phys. Lett. 51 (1987) 913.

[2] C. Adachi, T. Tsutsui, S. Saito, Appl. Phys. Lett. 57 (1990) 531.

[3] Y. Shirota, J. Mater. Chem. 10 (2000) 1.

[4] U. Mitschke, P. Baeuerle, J. Mater. Chem. 10 (2000) 1471

[5] S. A. VanSlyke, C. H. Chen, C. W. Tang, Appl. Phys. Lett. 69 (1996) 2160.

[6] M. Thelakkat, H.-W. Schmidt, Adv. Mater. 10 (1998) 219.

[7] K. Katsuma, Y. Shirota, Adv. Mater. 10 (1998) 223.

[8] H. Tanaka, S. Tokito, Y. Taga, A. Okada, Chem. Commun. (1996) 2175.

[9] M. Ichikawa, K. Hibino, N. Yokoyama, T. Miki, T. Koyama, Y. Taniguchi, Synth. Met. $156(2006) 1383$.

[10] Y.-L. Liao, W.-Y. Hung, T.-H. Hou, C.-Y. Lin, K.-T. Wong, Chem. Mater. 19 (2007) 6350.

[11] C.-Y. Lin, Y.-M. Chen, H.-F. Chen, F.-C. Fang, Y.-C. Lin, W.-Y. Hung, K.-T. Wong, R. C. Kwong, S. C. Xia, Org. Electron. 10 (2009) 181.

[12] Z. Jiang, Z. Liu, C. Yang, C. Zhong, J. Qin, G. Yu, Y. Liu, Adv. Funct. Mater. 19 (2009) 3987.

[13] C. Adachi, T. Tsutsui, S. Saito, Appl. Phys. Lett. 55 (1989) 1489.

[14] Y. Hamada, C. Adachi, T. Tsutsui, S. Saito, Jpn. J. Appl. Phys. 31 (1992) 1812.

[15] K. Tamao, M. Uchida, T. Izumizawa, K. Furukawa, S. Yamaguchi, J. Am. Chem. Soc. 118 (1996) 11974.

[16] M. Uchida, T. Izumizawa, T. Nakano, S. Yamaguchi, K. Tamao, K. Furukawa, Chem. Mater. 13 (2001) 2680.

[17] J. Bettenhausen, P. Strohriegl, Adv. Mater. 8 (1996) 507.

[18] S. B. Heidenhain, Y. Sakamoto, T. Suzuki, A. Miura, H. Fujikawa, T. Mor, S. Tokito, Y. Taga, J. Am. Chem. Soc. 122 (2000) 10240.

[19] G. Hughes, M. R. Bryce, J. Mater. Chem. 15 (2005) 94.

[20] Y.-J. Pu, M. Yoshizaki, T. Akiniwa, K.-i. Nakayama, J. Kido, Org. Electron. 10 (2009) 877.

[21] H. Sasabe, E. Gonmori, T. Chiba, Y.-J. Li, D. Tanaka, S.-J. Su, T. Takeda, Y.-J. Pu, K.-i. Nakayama, J. Kido, Chem. Mater. 20 (2008) 5951. 
[22] H. Tsuji, K. Sato, Y. Sato, E. Nakamura, J. Mater. Chem. 19 (2009) 3364.

[23] L. Na, W. Pengfei, L. Shiu-Lun, L. Weimin, L. Chun-Sing, L. Shuit-Tong, L. Zengtao, Adv. Mater. 22 (2010) 527.

[24] R. G. Kepler, P. M. Beeson, S. J. Jacobs, R. A. Anderson, M. B. Sinclair, V. S. Valencia, P. A. Cahill, Appl. Phys. Lett. 66 (1995) 3618.

[25] M. A. Baldo, D. F. O'Brien, Y. You, A. Shoustikov, S. Sibley, M. E. Thompson, S. R. Forrest, Nature 395 (1998) 151.

[26] M. Ichikawa, T. Kawaguchi, K. Kobayashi, T. Miki, K. Furukawa, T. Koyama, Y. Taniguchi, J. Mater. Chem. 16 (2006) 221.

[27] M. Ichikawa, N. Hiramatsu, N. Yokoyama, T. Miki, S. Narita, T. Koyama, Y. Taniguchi, Phys. Status Solidi (RRL) 1 (2007) R37.

[28] M. Ichikawa, S. Fujimoto, Y. Miyazawa, T. Koyama, N. Yokoyama, T. Miki, Y. Taniguchi, Org. Electron. 9 (2008) 77.

[29] S.-J. Su, T. Chiba, T. Takeda, J. Kido, Adv. Mater. 20 (2008) 2125.

[30] N. Matsumoto, C. Adachi, The Journal of Physical Chemistry C 114 (2010) 4652.

[31] M. A. Baldo, S. Lamansky, P. E. Burrows, M. E. Thompson, S. R. Forrest, Appl. Phys. Lett. 75 (1999) 4.

[32] Y. Han, et al., Journal of Physics D: Applied Physics 40 (2007) 1363.

[33] H. Murata, G. G. Malliaras, M. Uchida, Y. Shen, Z. H. Kafafi, Chem. Phys. Lett. 339 (2001) 161.

[34] S. Tabatake, S. Naka, H. Okada, H. Onnagawa, M. Uchida, T. Nakano, K. Furukawa, Jpn. J. Appl. Phys. 41 (2002) 6582. 
Figure Captions

Figure 1. Chemical structures of BpyB and TpyB.

Figure 2. DSC curves of BpyB and TpyB during first and second heating procedures.

Figure 3. (a), (b) Lowest-energy and third-lowest-energy computational optimized geometries, respectively, of BpyB. (c) Energy profile of the conformation change between these two geometries. Another local minimum exists at a dihedral angle of $30^{\circ}$. The structure of the secondlowest-energy geometry is close to that of (a).

Figure 4. (a) UV and visible absorption spectra (left axis) and PL spectra (right axis) of thin films of BpyB (thickness $77 \mathrm{~nm}$ ) and TpyB (thickness $88 \mathrm{~nm}$ ). (b) Phosphorescence spectra of meTHF solutions of the materials at liquid-nitrogen temperature (77 K). Weak bands around 400-425 nm are assigned to phosphorescence from the stabilizer contained in meTHF, 3,5-di-tert-butyl-4hydroxytoluene (BHT).

Figure 5. $J-V-L$ characteristics of OLEDs with BpyB or TpyB as electron-transporting materials and of a reference OLED with Alq: (a) Current density as a function of voltage $(J-V)$; (b) Luminous current efficiency as a function of current density $(L-J)$.

Figure 6. OLED device: (top) Device structure; (bottom) Chemical structures of two of the materials used in the device.

Figure 7. $J-V-L$ performance characteristics of phosphorescent OLEDs with BpyB or TpyB as dual-function hole-blocking and electron-transporting materials and of a reference OLED with the conventional structure: (a) Current density as a function of voltage $(J-V)$; (b) External quantum efficiency $(\mathrm{QE})$ as a function of current density.

Figure 8. Phosphorescent OLED device: (top) Device structure; (bottom) Chemical structures of three of the materials used in the device.

Figure 9. J-V-external QE characteristics of phosphorescent OLEDs with BpyB or TpyB as dualfunction hole-blocking and electron-transporting materials and of a reference OLED with the 
conventional structure: (a) Blue-light-emitting phosphorescent OLED with FIrpic as the dopant; (b) Red-light-emitting phosphorescent OLED with BtpIracac as the dopant. Device structures are the same as shown in Fig. 8 except for the dopants.

Figure 10. Electron transient photocurrent for time-of-flight measurements of BpyB at applied voltages, to the $\mathrm{Al}$ cathode, of 350,450 , and $550 \mathrm{~V}$. The dashed lines in green are for guides to determine the transit time of the photocurrent at $550 \mathrm{~V}$ and the three short red arrows represent each transit time. The inset shows the structure of the OLED device used.

Figure 11. Electron mobility as a function of electric field for BpyB, TpyB, and Alq. Data for Alq are from Murata (refer text).

Table 1. Thermal and electronic properties of BpyB and TpyB. 

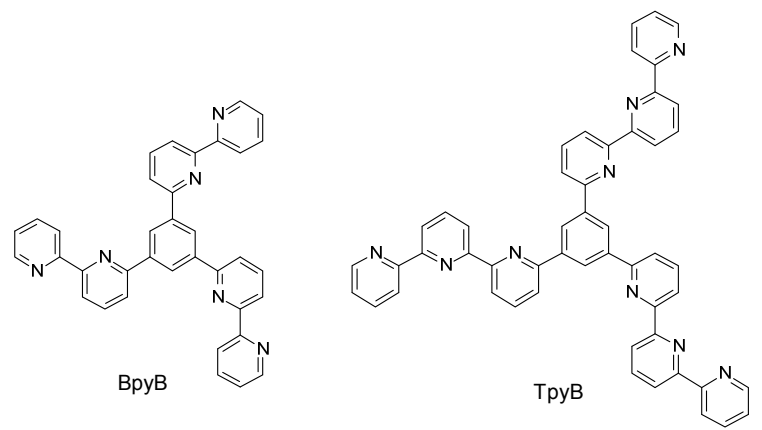

Figure 1. Chemical structures of BpyB and TpyB. 


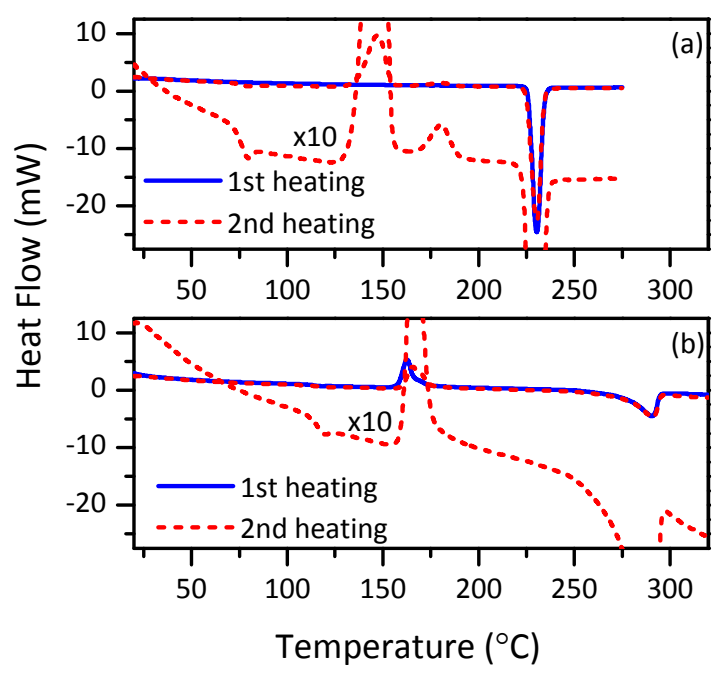

Figure 2. DSC curves of BpyB and TpyB during first and second heating procedures. 
(a)

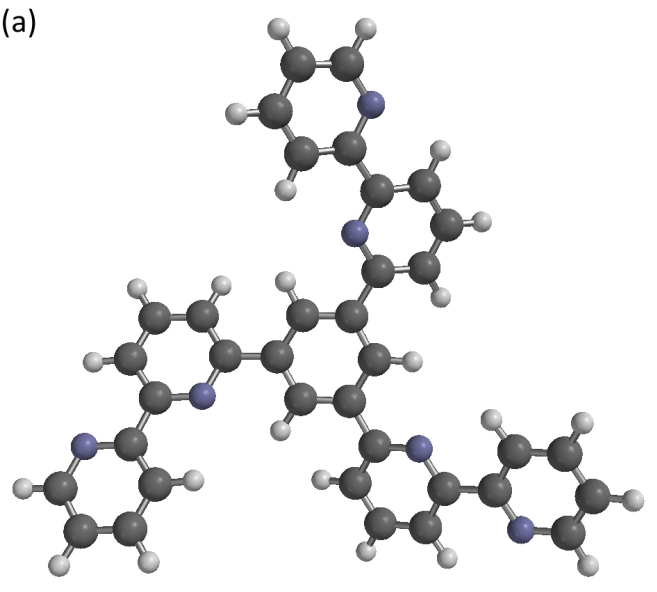

(b)

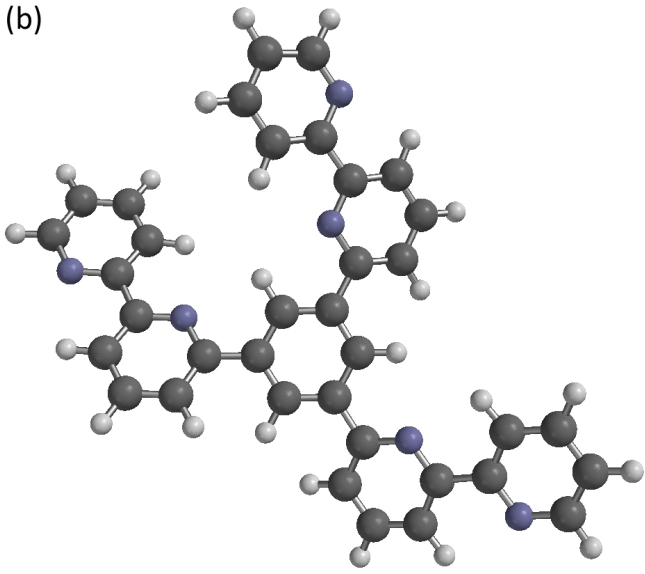

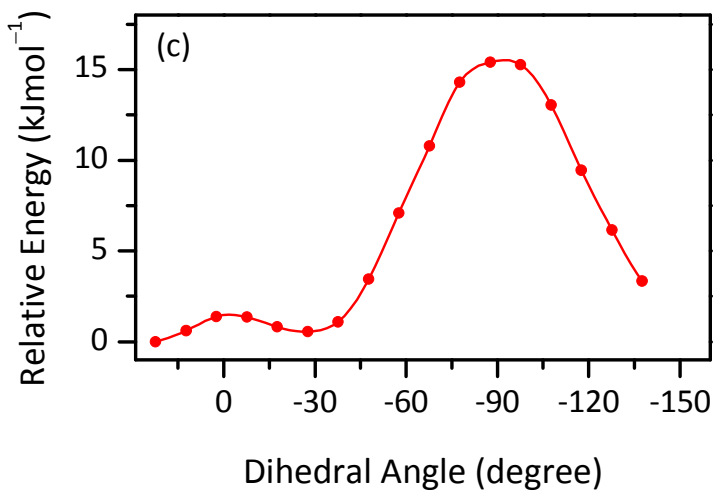

Figure 3. (a), (b) Lowest-energy and third-lowest-energy computational optimized geometries, respectively, of BpyB. (c) Energy profile of the conformation change between these two geometries. Another local minimum exists at a dihedral angle of $30^{\circ}$. The structure of the secondlowest-energy geometry is close to that of (a). 

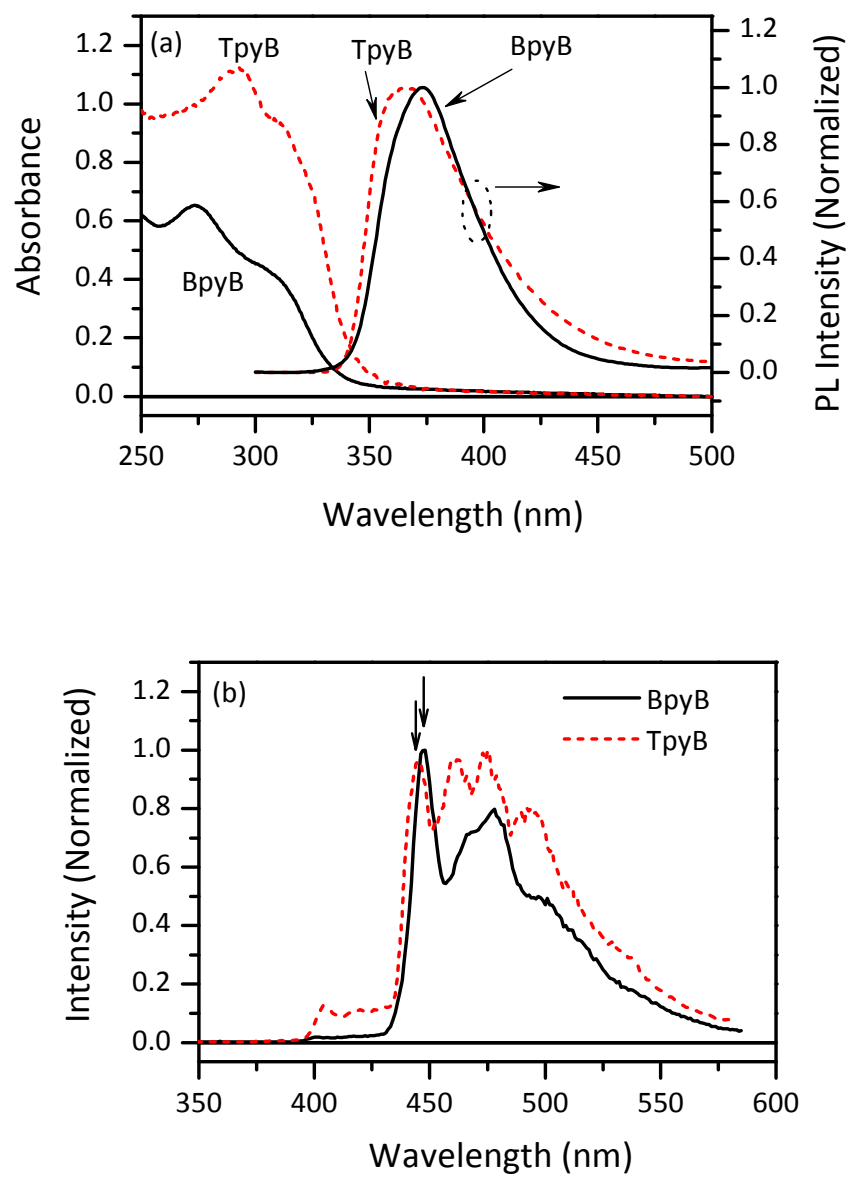

Figure 4. (a) UV and visible absorption spectra (left axis) and PL spectra (right axis) of thin films of BpyB (thickness $77 \mathrm{~nm}$ ) and TpyB (thickness $88 \mathrm{~nm}$ ). (b) Phosphorescence spectra of meTHF solutions of the materials at liquid-nitrogen temperature $(77 \mathrm{~K})$. Weak bands around $400-425 \mathrm{~nm}$ are assigned to phosphorescence from the stabilizer contained in meTHF, 3,5-di-tert-butyl-4hydroxytoluene (BHT). 

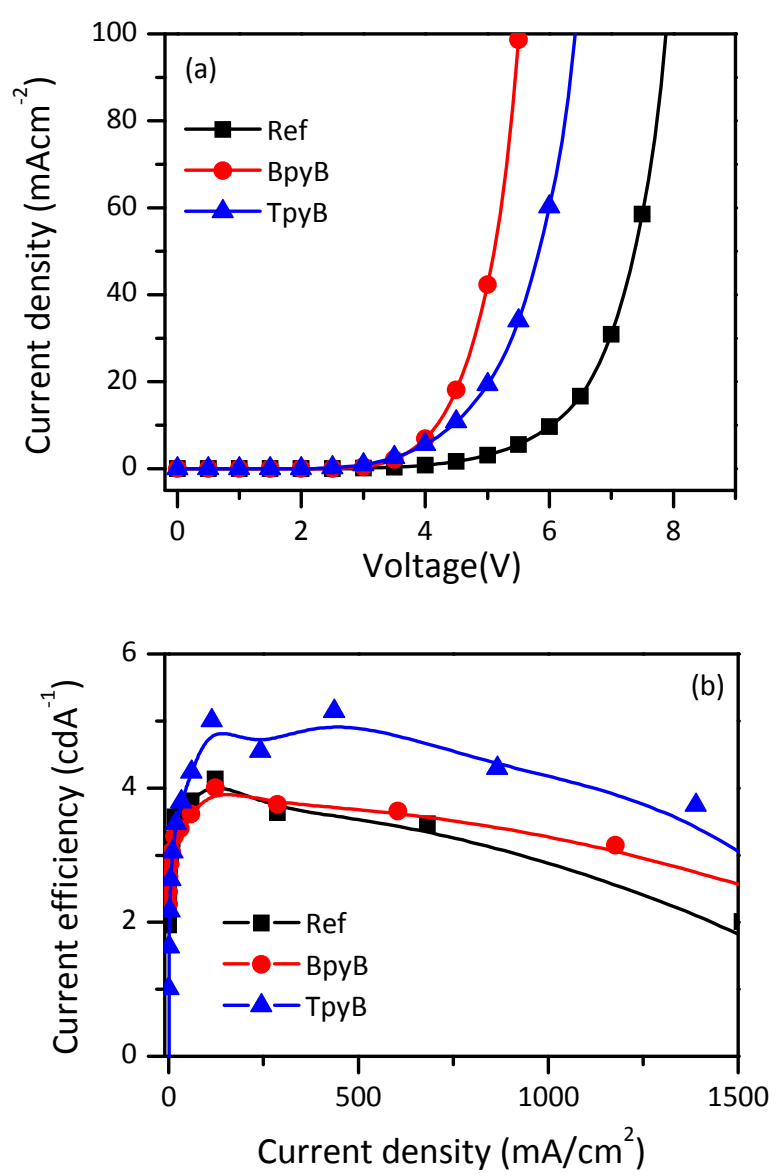

Figure 5. $J-V-L$ characteristics of OLEDs with BpyB or TpyB as electron-transporting materials and of a reference OLED with Alq: (a) Current density as a function of voltage $(J-V)$; (b) Luminous current efficiency as a function of current density $(L-J)$. 

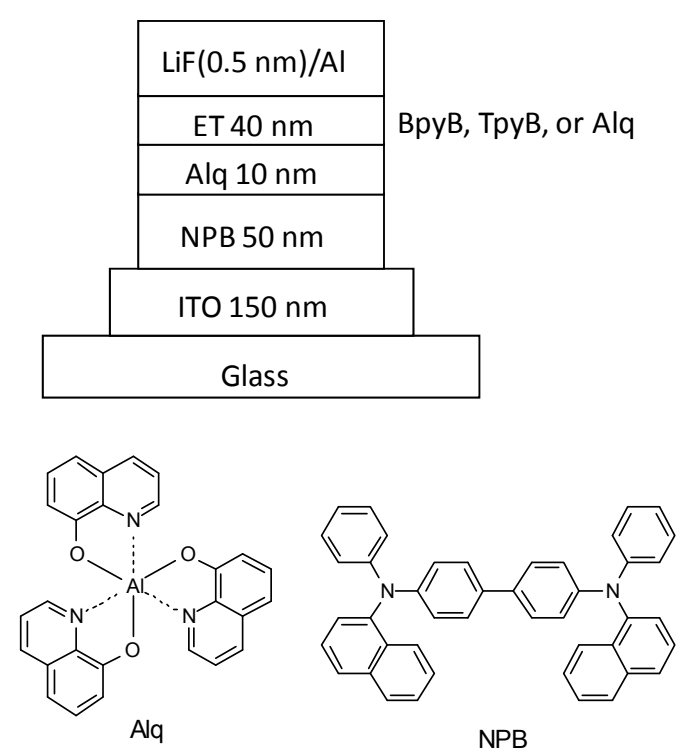

Figure 6. OLED device: (top) Device structure; (bottom) Chemical structures of two of the materials used in the device. 

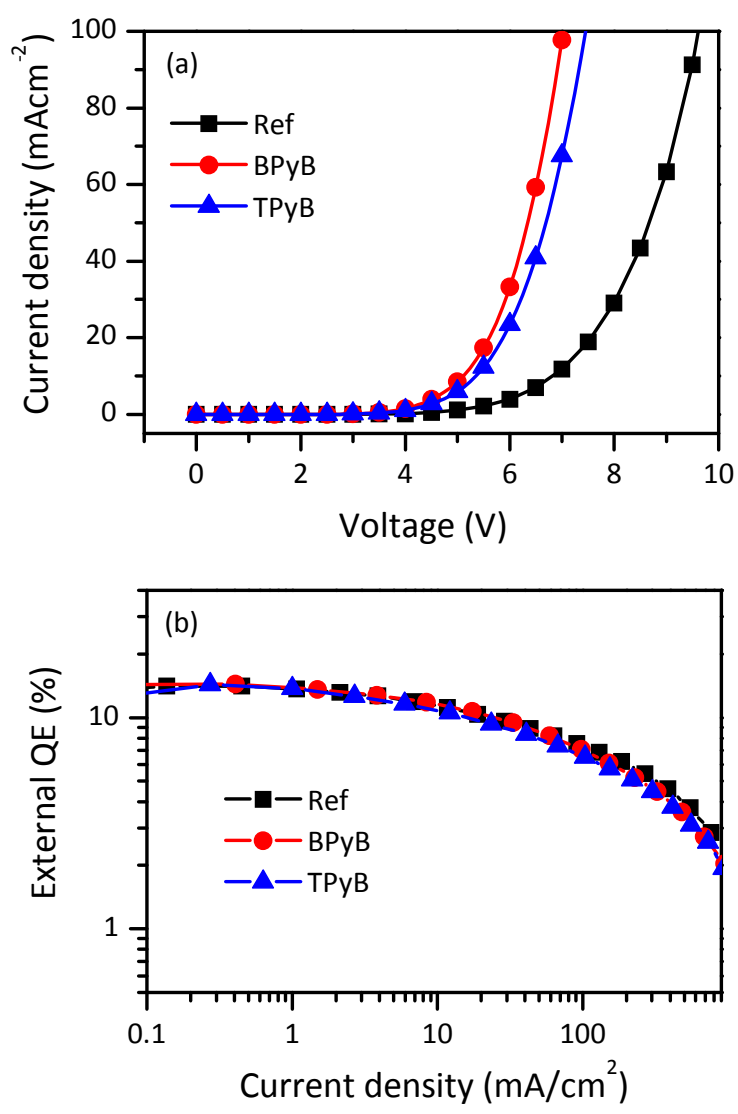

Figure 7. $J-V-L$ performance characteristics of phosphorescent OLEDs with BpyB or TpyB as dual-function hole-blocking and electron-transporting materials and of a reference OLED with the conventional structure: (a) Current density as a function of voltage $(J-V)$; (b) External quantum efficiency $(\mathrm{QE})$ as a function of current density. 

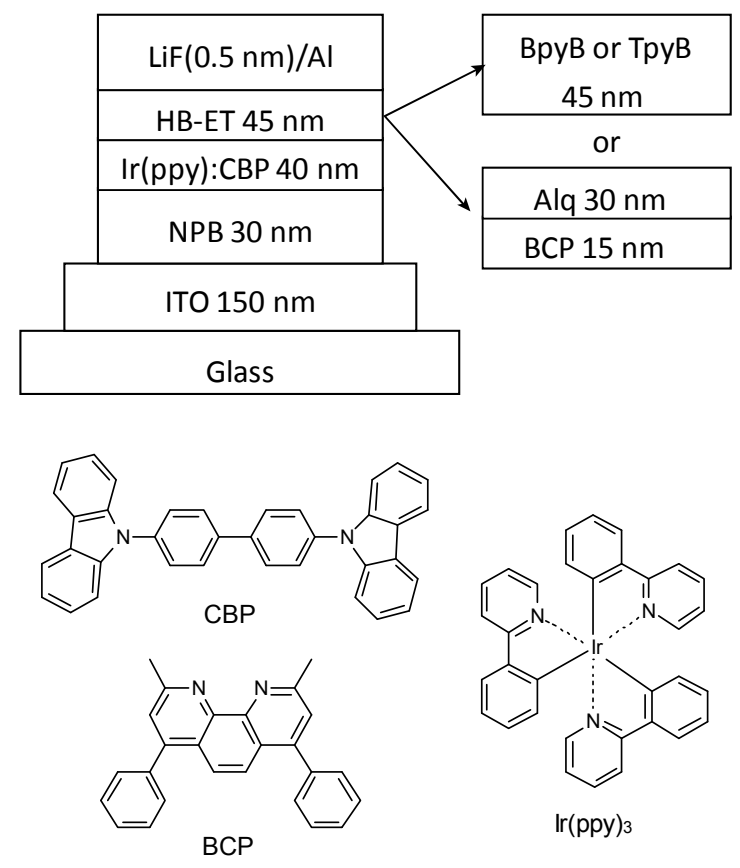

Figure 8. Phosphorescent OLED device: (top) Device structure; (bottom) Chemical structures of three of the materials used in the device. 


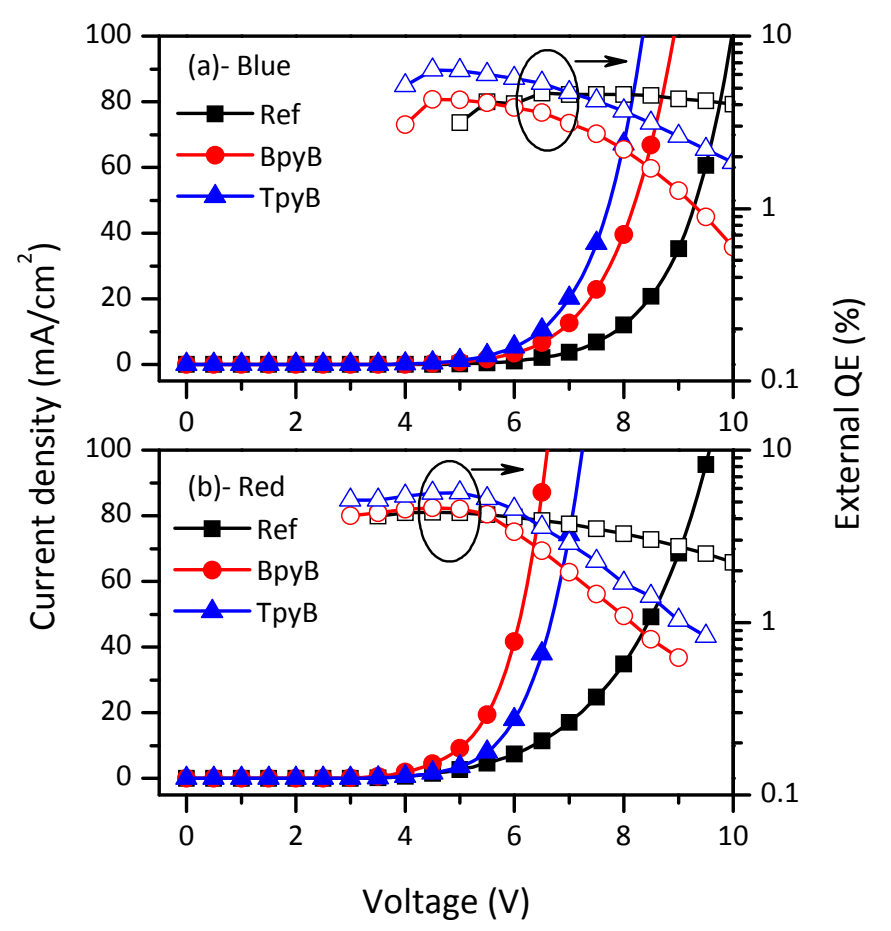

Figure 9. J-V-external QE characteristics of phosphorescent OLEDs with BpyB or TpyB as dualfunction hole-blocking and electron-transporting materials and of a reference OLED with the conventional structure: (a) Blue-light-emitting phosphorescent OLED with FIrpic as the dopant; (b) Red-light-emitting phosphorescent OLED with BtpIracac as the dopant. Device structures are the same as shown in Fig. 8 except for the dopants. 


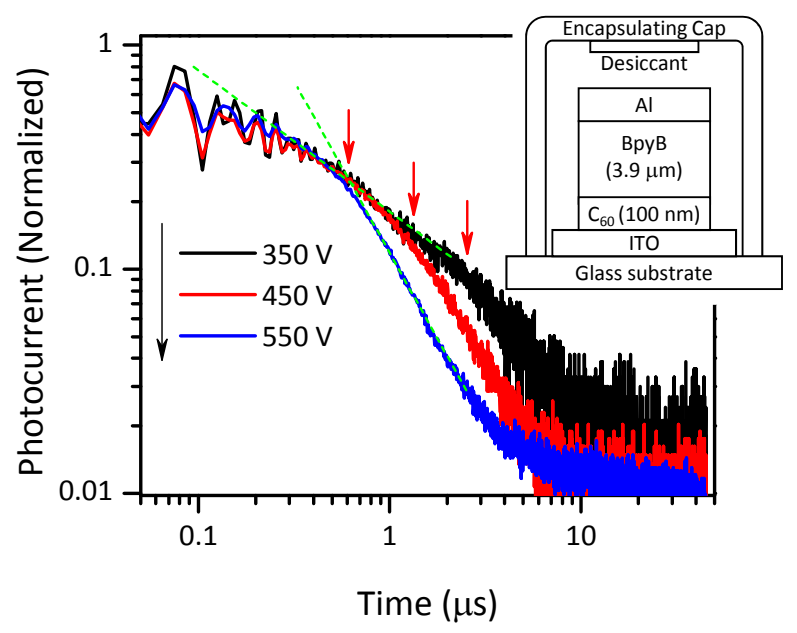

Figure 10. Electron transient photocurrent for time-of-flight measurements of BpyB at applied voltages, to the $\mathrm{Al}$ cathode, of 350,450 , and $550 \mathrm{~V}$. The dashed lines in green are for guides to determine the transit time of the photocurrent at $550 \mathrm{~V}$ and the three short red arrows represent each transit time. The inset shows the structure of the OLED device used. 


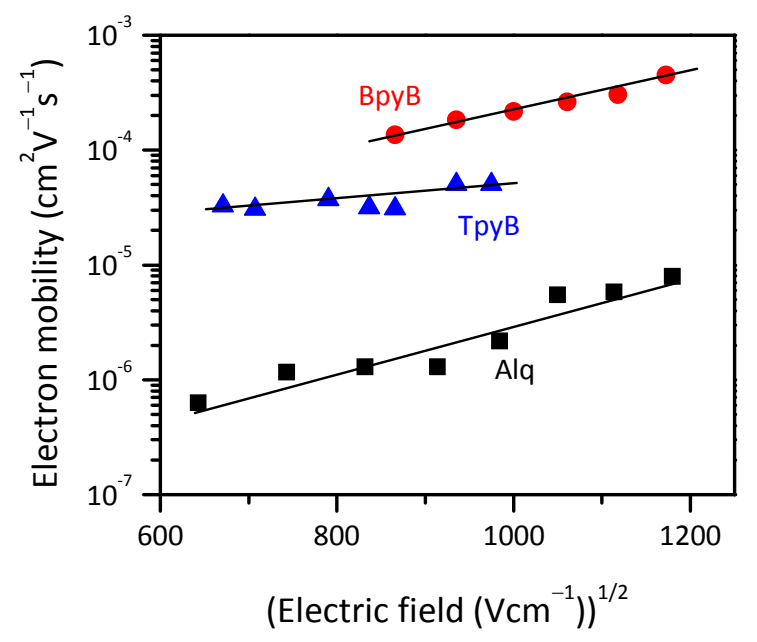

Figure 11. Electron mobility as a function of electric field for BpyB, TpyB, and Alq. Data for Alq are from Murata (refer text). 
Table 1. Thermal and electronic properties of BpyB and TpyB

\begin{tabular}{ccccccc}
\hline & $I \mathrm{p}(\mathrm{eV})$ & $E \mathrm{a}(\mathrm{eV})$ & $E \mathrm{~g}(\mathrm{eV})$ & $E \mathrm{~g}^{\mathrm{T}}(\mathrm{eV})$ & $T \mathrm{~m}\left({ }^{\circ} \mathrm{C}\right)$ & $T \mathrm{~g}\left({ }^{\circ} \mathrm{C}\right)$ \\
\hline \hline BрyB & 6.45 & 2.85 & 3.60 & 2.77 & 240 & 75 \\
TpyB & 6.60 & 3.00 & 3.60 & 2.79 & 305 & 110 \\
\hline
\end{tabular}

